\title{
sciendo
}

\section{Analysis of Polymorphic Variants of the Dopamine Transporter (DAT1) Gene Polymorphism and Personality Traits Among Athletes}

\author{
by \\ Monika Michałowska-Sawczyn¹, Milena Lachowicz', Anna Grzywacz², \\ Aleksandra Suchanecka², Jolanta Chmielowiec ${ }^{3}$, Krzysztof Chmielowiec ${ }^{3}$, \\ Jakub Chycki ${ }^{4}$, Grzegorz Trybek ${ }^{5}$, Piotr Żmijewski ${ }^{6}$, Paweł Cięszczyk ${ }^{1}$
}

Personality traits, especially in sport are modulatory factors of athletes' behavior - his/ her conscientiousness, the will to achieve an aim, perseverance and motivation of activity. Not only are biological predispositions related to anatomical or biochemical traits of success, but they are also largely determined by personality traits that result from genetic factors. In our research we joined tests of athlete's personality in correlation with genotypes of the dopamine transporter (DAT1) gene polymorphism. The selection of this polymorphism was based on previous reports connecting the influence of dopamine with motivation and numerous arguments supporting its correlation with human behavior. We observed significant differences among polymorphisms DAT 9/9, 9/10, 10/10 in terms of proportion of particular genotypes between athletes and the control group. We also found significant differences in the NEO FFI sten scale for conscientiousness. We noticed that anxiety was related with genotypic variants of DAT1, specifically the 9/10 VNTR variant, which conditioned lower levels of anxiety in the group of tested athletes. By contrast, the lower sten value of agreeability was statistically significant for the group of athletes that were carriers of the 10/10 VNTR genotype. Heterozygous 9/10 VNTR among athletes showed lower levels of anxiety in comparison with the control group, whereas agreeability determined using the NEO FFI scale represented a lower value among athletes that had the 10/10 polymorphism. We may thus conclude that the presence of polymorphic variants of the dopamine transporter gene corresponds to athletes' personality traits.

Key words: dopamine, personality, athletes.

\section{Introduction}

Who we are, how we behave in different life situations frequently determines our path to success or failure. Personality traits, especially in sport are modulatory factors of athlete's behavior - his/her conscientiousness, the will to achieve an aim, perseverance and motivation of activity. The personality is determined by both the environmental and genetic factors, and these factors in different proportions explain athletic behavior. Variables such as age, gender, race and the culture of the examined individual, do not influence essential personality traits that are biologically determined and important for human adaptation (McCrae and Costa, 1985). The NEO-

\footnotetext{
1 - Faculty of Physical Culture, Gdańsk University of Physical Education and Sport, Gdańsk, Poland.

2 - Independent Laboratory of Health Promotion of the Pomeranian Medical University in Szczecin, Szczecin, Poland

3 - Department of Hygiene and Epidemiology, Collegium Medicum, University of Zielona Góra, Poland.

4 - Institute of Sport Sciences, The Jerzy Kukuczka Academy of Physical Education in Katowice, Poland.

5 - Department of Oral Surgery, Pomeranian Medical University, Szczecin, Poland.

6 - Faculty of Physical Education, Józef Piłsudski University of Physical Education in Warsaw, Poland.
} 
Five Factor Inventory (NEO-FFI) is applied to evaluate elementary personality traits (McCrae and Costa, 1985).

A dimensional structure of personality can be reflected using the five-factor model, which is the hierarchical model, with specific personality traits corresponding with each trait dimension, i.e. extraversion, neuroticism, openness, agreeableness and conscientiousness. In the spectrum of the extraversion dimension, two distinct types can be distinguished: introverted (unsociable, quiet, and passive) and extraverted (sociable, outgoing, and active). The neuroticism dimension distinguishes between emotional stability (calm, controlled, and even-tempered) and emotional instability (anxious, hostile, and irritable). Openness distinguishes between those who are open to new experiences (curious, creative, and imaginative) and those who like the familiar (conventional, uncreative, and unimaginative). The agreeableness dimension extends from traits associated with being compassionate (good-natured, unselfish, and forgiving) to those which are antagonistic (cynical, rude, and uncooperative). The fifth dimension is conscientiousness, which distinguishes those who are conscientious (organized, punctual, and hardworking) from those who are lackadaisical (unreliable, lazy, and careless).

When considering twin and family studies, variance in personality traits is estimated to be in the range of $30-60 \%$ when analyzing heritable components of an individual (Bouchard, 1994).

Most research in the area of competitive sport concentrates mainly on searching for personality differences within diverse groups of athletes and non-athletes. Current research indicates higher levels of extraversion and lower levels of neuroticism among athletes compared to a normative sample (Hughes et al., 2003).

In this research, we tested athlete personality traits in correlation with the genotype of the dopamine transporter (DAT1) gene polymorphism. The selection of this polymorphism was based on previous reports connecting the influence of dopamine with motivational systems and numerous arguments supporting its correlation with human behavior.

The purpose of this study was to determine whether personality traits had an impact on athletic success. Current literature indicates inconsistent patterns between personality dimensions and athletic performance. This seems to run counter to the patterns of other performance-related domains, namely academic and organizational settings, where personality (and in particular conscientiousness) has been considered to be the element directly influencing success (Ozer and Benet-Martinez, 2006; Krokosz and Jachimek, 2018).

The relationship between higher-order personality dimensions and sport-related coping behavior is not well understood. Numerous studies connect coping behavior with the five dimensions of personality, however, little has been studied in the domain of competitive sport (Carver and Connor-Smith, 2010). The authors hypothesized that higher levels of extraversion and conscientiousness could be linked with more problem-focused coping (coping directed towards resolving the problem itself, such as expending more effort, seeking support, and re-analysing past experiences), whereas higher levels of neuroticism would be associated with both emotion-focused coping (coping by ventilating, managing or palliating emotions) and avoidance coping (removing oneself mentally or physically from the stressor).

Diverse brain functions are regulated by dopamine, the hormone that also plays a role in numerous mental conditions (Grace, 2016). Dopamine is a hormone which function is neurotransmission in many behavioral and decision-making processes which include: aggression, sexual behavior, reward, learning, and memory. One of the elements critical for dopamine homeostatsis is the dopamine transporter (DAT), which is responsible for dopamine reuptake in the striatum (Sesack et al., 1998). The DAT encoding gene, that spans over 60 $\mathrm{kb}$ and consists of 15 exons and 14 introns, is situated on chromosome 5 (5p.15.32). However, the protein-coding component of DAT1 excludes exon 1 and terminates within exon 15 with a single transcription start site, as determined by 5RACE and RNase protection assays (Kawarai et al., 1997).

A DAT1 3'-untranslated region (UTR) VNTR (variable number tandem repeat) has been the focus of multiple research groups in an 
attempt to determine an association between the VNTR and several CNS disorders. The polymorphism consists of a 40-base pair tandem repeat which is typically observed as $3-11$ copies with alleles consisting of 9 or 10 repetitions representing the most common genotypes. Although the VNTR is not located within the exonic regions or splice sites of DAT1, it is established that polymorphisms in noncoding loci may result in differing gene expression through a variety of mechanisms, including changes in RNA stability, transport, and subsequent translation (VanNess et al., 2005). A multitude of in vitro functional assays have been undertaken in order to elucidate the potential influence of VNTRs on gene expression. Functional analysis, using the luciferase assay reporter system, demonstrated that allelic variants of the DAT1 VNTR altered luciferase expression, but luciferase expression was not significantly different between the VNTR 9- and 10-repeat alleles (Greenwood and Kelsoe, 2003). Such inconsistent observations continued as VanNess et al. (2005) utilized a combination of binding assays, immunoblots, and stable cell line transfection. It was deduced that HEK293 cells transfected with the 10-repeat allele exhibited notably greater DAT density compared to the 9repeat allele (VanNess et al., 2005).

Hence, in the present research we concentrated on personality traits in connection with polymorphic variants DAT1 VNTR among athletes in a case control analysis.

\section{Methods}

\section{Participants}

The study was conducted among 200 Polish healthy (no prior history of substance dependency or psychosis) male combat athletes aged $22.9 \pm 4.2$ (judo, $\mathrm{n}=51$; wrestling, $\mathrm{n}=38$; boxing, $\mathrm{n}=50$, kickboxing, $\mathrm{n}=32$; karate, $\mathrm{n}=29$ ). All of them were ranked in the top 10 nationally in their respective weight category. The study population included 16 athletes classified as 'topelite' (gold medalists in the World and European Championships, World Cups or Olympic Games) and 68 athletes classified as 'elite' (silver or bronze medalists in the World and European Championships, World Cups or Olympic Games). The others $(n=69)$ were classified as 'sub-elite' (participants in international competitions, with no less than 8 years of training experience).
Various methods were used to obtain the samples, including targeting national teams and providing information to national coaching personnel and athletes attending training camps.

Controls included 102 healthy (nondependent and non-psychosis) Polish male volunteers aged $21.6 \pm 2.6$. All athletes and controls were Caucasian to reduce the possibility of racial gene skewing and to overcome any potential problems due to population stratification.

Methods

The NEO Five-Factor Personality Inventory (NEO-FF) and the State-Trait Anxiety Inventory (STAI) questionnaires were applied in testing the group of athletes, as well as the control group. The sten scale was used to present the results of NEO-FFI and STAI tests. To analyze the history of substance dependence and psychosis, the medical records and author's own survey were applied. Venous blood drawn from the elbow vein was the source of DNA used in the analysis.

Anxiety is frequently evaluated with the use of the Anxiety Inventory (STAI) questionnaire. The anxiety state is mainly determined as the conditioned and transient psychological state of an individual. Moreover, it is a relatively constant personality trait. Two independent subscales, containing 20 statements each, were elements of the STAI questionnaire. One of the subscales measured the anxiety trait $(X-1)$, whereas the other measured the motivation trait (X-2). Personality traits defined with the Big Five model (neuroticism, extraversion, openness, conscientiousness and agreeableness) are the components that can be diagnosed on the basis of the NEO Five Factor Inventory questionnaire, that includes 60 statements (of a self-reported nature), requiring respondents to incorporate a research approach in assessing themselves.

Tubes with EDTA (anticoagulant) were used to collect blood for the genetic assays. Genomic DNA from blood leukocytes was obtained with the use of a High Pure Polymerase Chain Reaction (PCR) Template Preparation extraction kit (Roche Diagnostics, Mannheim, Germany). The process of extraction was conducted in accordance with manufacturer's instructions. Such extracted samples of DNA were stored at $4^{\circ} \mathrm{C}$ until further analysis. 
Venous blood collected according to standard procedures was the source of genomic DNA. The PCR method was used to genotype the samples. The DAT1 genotypes were grouped according to the presence of the 9 or 10 repeat variants. Genotyping was performed using the PCR-VNTR method, with primers: F: 50-TGT GGT GTA GGG AAC GGC CTG Ag 30, R: 50-CTT CCT GGA GGT CAC GGC TCA AGG 30; in the final volume of $25 \mathrm{~L}$ PCR mix per reaction, with $100 \mathrm{ng}$ genomic DNA, 10 pmol of primers, $50 \mathrm{mM}$ $\mathrm{KCl}, 10 \mathrm{mM}$ TrisHCl, $1.5 \mathrm{mM} \mathrm{MgCl} 2,200 \mathrm{M} \mathrm{dATP}$, dCTP, dTTP, dGTP and $0.8 \mathrm{U}$ of the Tag polymerase. Conditions for reaction were as follows: 5 min of initial denaturation in $94 \mathrm{C}, 55 \mathrm{~s}$ of denaturation in $94 \mathrm{C}, 50 \mathrm{~s}$ of primer hybridization in $55 \mathrm{C}$ and $1 \mathrm{~min}$ of elongation in $72 \mathrm{C}$, repeated in 30 cycles, $10 \mathrm{~min}$ of final elongation in $72 \mathrm{C}$. The amplified products were visualized using ethidium bromide stained gel electrophoresis ( $3 \%$ agarose) and UV photography. The products' lengths were $450 \mathrm{bp}$ for 10 repeat alleles and $410 \mathrm{bp}$ for 9 repeat alleles.

\section{Statistical analysis}

Concordance between the genotype frequency distribution and Hardy-Weinberg equilibrium (HWE) was tested with HWE software (http://www.oege.org/software/hwe-mrcalc.html). The relationships between DAT variants, elite athletes and control participants and the NEO Five Factor Inventory (NEO-FFI) were analyzed using a multivariate analysis of Factor effects ANOVA (NEO-FFI/ scale STAI/ $\times$ genetic feature $\times$ control and elite athletes $\times$ (genetic feature $\times$ control and elite athletes)). The condition of homogeneity of variance was satisfied (Levene test; $p>0.05$ ) and distributions of dependent variables close to normal were satisfied. DAT genotype frequencies between healthy control participants and elite athletes were tested using the chi square test. All computations were performed using STATISTICA 13 (Tibco Software Inc, Palo Alto, CA, USA) for Windows (Microsoft Corporation, Redmond, WA, USA).

\section{Results}

The observed DAT polymorphism frequencies did differ from expectations based on the Hardy-Weinberg theorem in elite athletes, but they did not differ in the control group.
In comparison with the control group, statistically significant difference in the genotype frequency for the DAT gene in elite athletes was found (9/9 0.04 vs. $9 / 90.08,9 / 100.48$ vs. 9/10 0.33, $10 / 100.48$ vs. $10 / 100.59, \chi 2=6.79, p=0.033$ ) as well as a difference in the frequency for the DAT gene between athletes and the control group, which was not statistically significant $(90.28$ vs. 9 $0.25,100.72$ vs. $100.75, \chi 2=0.84, p=0.359$ ) (Table 1).

The results of the $2 \times 3$ factorial ANOVA of the NEO Five-Factor Personality Inventory (NEO-FFI) and the State-Trait Anxiety Inventory (STAI) sten scales are summarized in Table 4 . We found a significant result when comparing groups (elite athletes vs. controls) for Conscientiousness ( $\mathrm{F}_{1,296}=$ $20.81, p=0.000)$, accounting for $6.6 \%$ of variance. In addition to those findings, we found group $\mathrm{x}$ $D A T$ genotype interactions: for STAI ST (Figure 1, $\left.\mathrm{F}_{2,296}=3.55, p=0.03\right)$, Neuroticism $\left(\mathrm{F}_{2,296}=6.95, p=\right.$ 0.001 ), Agreeability (Figure 2, $\mathrm{F}_{2,296}=6.44, p=$ $0.002)$ and Conscientiousness $\left(\mathrm{F}_{2,296}=8.14, p=\right.$ 0.0004 ) responsible for $2.3 \%, 4.5 \%, 4.2 \%$ and $5.2 \%$ phenotypic variation, respectively. Post-hoc analysis is shown in Table 5.

\section{Discussion}

In our research, we observed distinct and significant differences in the DAT 9/9, 9/10, 10/10 polymorphism in the proportion of particular genotypes between the athlete group and the control group (Table 1). Significant differences were also observed in the sten scale NEO FFI for conscientiousness, i.e., 7.18 vs. $7.59(p=0.002)$ for athletes and controls, respectively (Table 2 ).

Moreover, we noticed that anxiety was connected with genotypic variants of DAT1, which is shown in Table 3 and Figure 1. The genotypic variant 9/10 VNTR, which conditions lower levels of anxiety, was shown to be significant in this analysis among the group of athletes. However, a lower stent value of agreeability was statistically significant for athletes that were carriers of the 10/10 VNTR genotype. The correlation is presented in Table 3 and Figure 2.

According to our results, athletes that were heterozygotes 9/10 VNTR demonstrated lower levels of anxiety than the control group, whereas for NEO FFI agreeability, we observed lower values for athletes who represented the 
10/10 polymorphism.

Motivation processes are connected with striatal dopaminergic activity and there are few elements indicating a significant role of repeat polymorphisms in DAT genes. One of the examples show that carriers of DAT1 9-repeat represent more striatal activity when processing a monetary reward (Dreher et al., 2009), thereby suggesting increased reward sensitivity in comparison with 10-repeat carriers.

Table 1

Frequency of genotypes and alleles of the DAT1 gene polymorphisms in athletes and controls DAT

\begin{tabular}{lccccc} 
& \multicolumn{3}{c}{ Genotypes } & \multicolumn{3}{c}{ Alleles } \\
\cline { 2 - 6 } & $9 / 9$ & $9 / 10$ & $10 / 10$ & 9 & 10 \\
Group & $\mathrm{N}(\%)$ & $\mathrm{N}(\%)$ & $\mathrm{N}(\%)$ & $\mathrm{N}(\%)$ & $\mathrm{N}(\%)$ \\
Athletes & 8 & 96 & 96 & 112 & 288 \\
$\mathrm{~N}=200$ & $(0.04)$ & $(0.48)$ & $(0.48)$ & $(0.28)$ & $(072)$ \\
& & & & & \\
Control & 8 & 34 & 60 & 50 & 154 \\
$\mathrm{~N}=102$ & $(0.08)$ & $(0.33)$ & $(0.59)$ & $(0.25)$ & $(0.75)$ \\
$\chi^{2}$ & & $\mathbf{6 . 7 9}$ & & \multicolumn{2}{c}{0.84} \\
p value & & $\mathbf{0 . 0 3 3}$ & & \multicolumn{2}{c}{0.359} \\
\end{tabular}

$p$ - statistical significance $\chi^{2}$ test, $N$ - number of participants

Significant between-group differences are marked in bold print.

Table 2

Differences in STAI and NEO Five Factor Inventory results between healthy control participants and athletes.

\begin{tabular}{lcccc}
\hline $\begin{array}{l}\text { STAI / NEO Five Factor } \\
\text { Inventory/ } \\
\text { (sten scale) }\end{array}$ & $\begin{array}{c}\text { Athletes } \\
(\mathrm{N}=200)\end{array}$ & $\begin{array}{c}\text { Control } \\
(\mathrm{N}=102)\end{array}$ & $p$ value & Cohen's d \\
& & & & \\
\hline STAI ST & $4.90 \pm 2.22$ & $4.73 \pm 2.14$ & .688 & .08 \\
STAI C & $5.28 \pm 2.37$ & $5.20 \pm 2.00$ & .848 & .04 \\
Neuroticism/scale & $4.84 \pm 2.30$ & $4.82 \pm 1.72$ & .967 & .01 \\
Extraversion/scale & $6.20 \pm 1.88$ & $6.14 \pm 1.78$ & .864 & .03 \\
Openness/scale & $4.42 \pm 1.62$ & $4.63 \pm 1.75$ & .538 & .12 \\
Agreeability/scale & $5.18 \pm 2.13$ & $5.51 \pm 1.88$ & .411 & .16 \\
Conscientiousness/scale & $\mathbf{7 . 1 8 \pm 1 . 9 2}$ & $\mathbf{5 . 9 8 \pm 1 . 7 9}$ & .002 & .65 \\
\hline
\end{tabular}

$p$ - statistical significance $t$-Student's test, $N$-number of participants Significant between-group differences are marked in bold print. 
Table 3

Differences in DAT and STAI /NEO Five Factor Inventory between healthy control subjects and athletes.

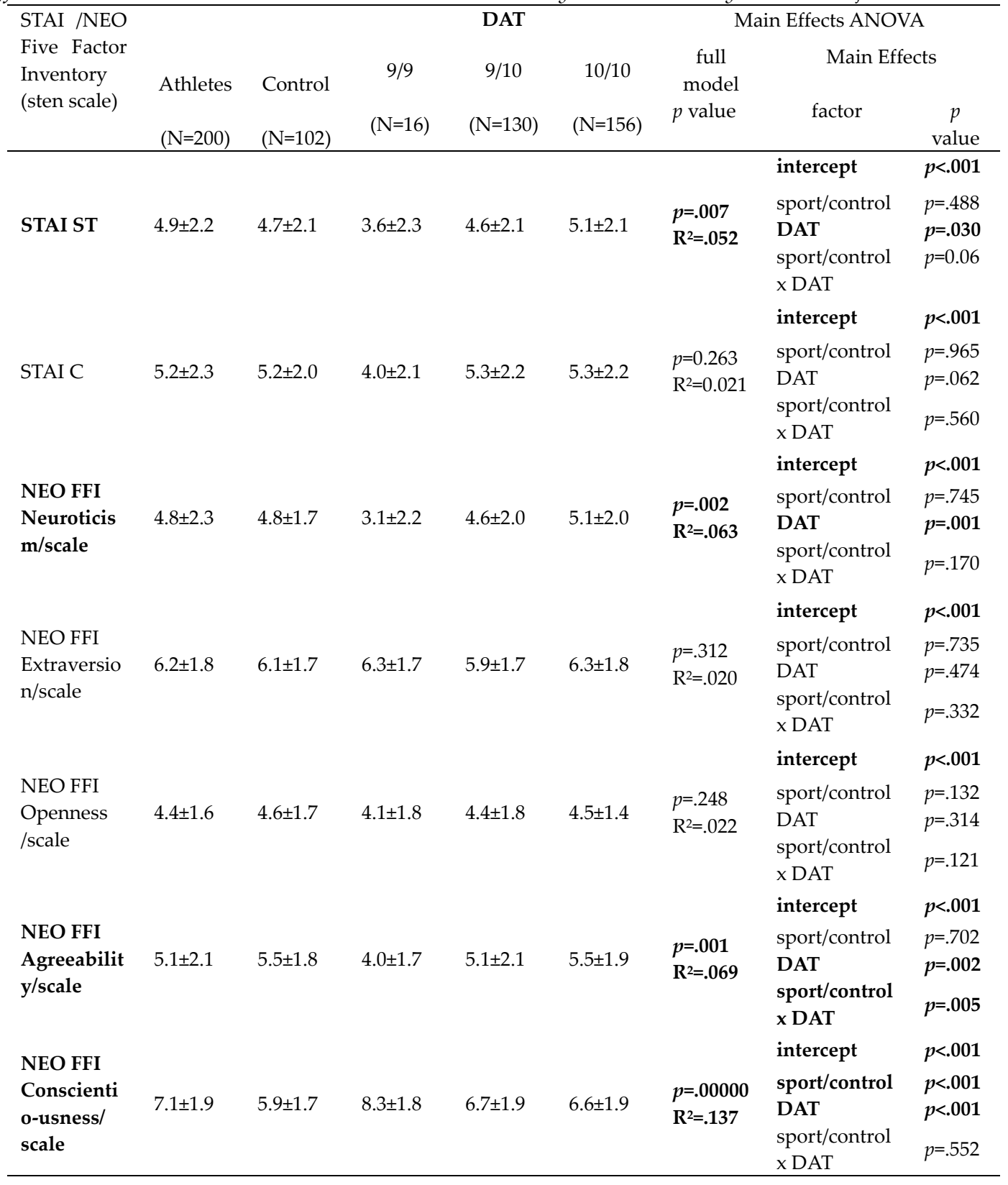


$F(2,296)=2.85, p=0.059$



Figure 1

The group (elite athletes vs. healthy controls) DAT polymorphism interaction for the STAI ST.

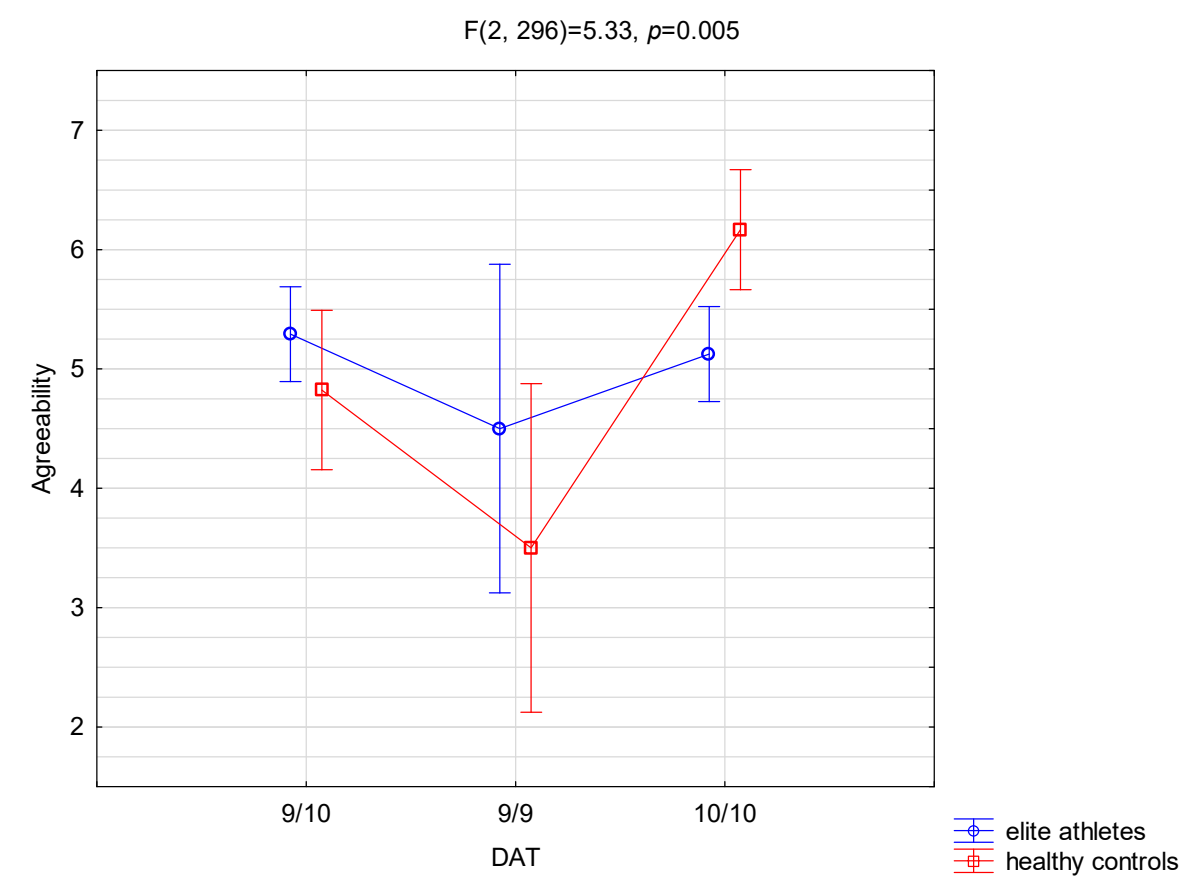

Figure 2

The group (elite athletes vs. healthy controls) $x$ DAT polymorphism interacti on for the NEO Five Factor Inventory scale of Agreeability. 
Motivational behavior is usually augmented in the event of increased rewardrelated activity and is connected with increased synaptic DA availability. In 2012, Enter et al. (2012) noticed that the DAT1 9-repeat carriers presented increased emotion-driven action tendencies in relation to stimuli that communicated motivation driven by the presence of an observer. It was based on the fact that during the experiment, participants were immediately rewarded with the appearance of happy faces or the shrinking/disappearance of angry faces in response to the speed with which individuals pushed or pulled the joystick, respectively. Similar were the observations made by Talmi et al. (2008). In their study a positive relationship between the vigor of action and motivation to obtain a reward was observed, which corresponded to an increased oxygen leveldependence signal in the Nucleus Accumebens, suggesting increased DA signaling. Interestingly, Enter also indicated that responses of those who carried the 9-repeat polymorphism were influenced by irrelevant social-emotional features, whereas responses of 10-repeat homozygotes did not show such patterns. However, there are other studies suggesting the existence of increased sensitivity to both positive and negative contextual conditions. According to Belsky and Pluess (2009), increased sensitivity in an individual leads not only to increased vulnerability to the negative effects of an adverse environment, but also to greater receptivity to beneficial outcomes in a positive context. In our research we also observed a positive association with anxiety as the genotype variant $9 / 10$ VNTR significantly conditioned lower anxiety in the tested group of athletes.

Statistical significance connected with the homozygotic variant $9 / 10$ and behavior pattern was noticed by Cherepkova et al. (2019) who suggested that the 9/10 genotype of DAT gene presented contradictory results to the relevant published data according to an analysis of a group of Russian males. The carriers of this genotype were less prone to commit violent crimes, based on the results of calculated odds ratios.

However, we should be very careful, when interpreting obtained results, because robust negative findings for striatal DAT availability are discursive in connection with previous findings that suggest positive association between DAT availability and cognitive functions in healthy individuals. Numerous results also suggest that subcortical SERT availability shows no association with neuropsychological functions and personality traits that are assessed. Burke et al. (2011) stated that neuropsychological and personality measurements in young healthy people suggested no association with subcortical SERT or striatal DAT availabilities in the brain.

When concentrating on the dopamine transporter, we observed the presence of the homozygous DAT genotype 9/9 at more than 5 times greater frequency in the group of elite athletes than in controls. Allele 9 occurred more than twice more often in elite athletes than in controls (Burke et al., 2011). When comparing the 10-repeat allele with the previously mentioned one, an association occurs with increased gene expression in both in vitro (Mill et al., 2002) and in vivo (Pasini et al., 2013). Nonetheless, the results as well as their interpretation are still discursive as there are also studies that suggest opposite allelic associations.

Self-control refers to the phenomenon of people overcoming their natural and automatic tendencies, desires, and behaviors, and resisting short-term temptations to achieve long-term goals. Self-control seems to be the core element for achieving optimal competitive performance. Neuroticism has a negative correlation with selfcontrol; agreeableness, extraversion, openness, and responsibility, are positively related to selfcontrol; self-control is a prerequisite for individuals to adapt to their social environment. In addition, researchers found differences in the relationship between extraversion, openness, and self-control; some studies found that extraversion and self-control had a significant negative correlation (Green et al., 2016), while others found extraversion was not significantly related with self-control (de Vries and van Gelder, 2013). In the same way, research on the relationship between openness and self-control has also produced inconsistent results.

Another aspect of our research included analysis of athletes' personality traits. Our results seem to correspond with previously described associations between significant difference occurring in the stent scale NEO FFI for 
conscientiousness in the group of athletes and controls; 7.18 vs. $5.98, p=0.002$, respectively.

Self-efficacy of individuals is influenced by personality traits. Studies have linked the Big Five traits and self-efficacy (Judge et al., 2007), for example, neuroticism is negatively correlated with self-efficacy, and extraversion, openness, agreeableness, and responsibility positively correlated with self-efficacy (Judge et al., 2007). Some scientists found that individuals with higher scores of conscientiousness had higher selfefficacy beliefs (Brown et al., 2011). Openness shifts perceptions of demands into challenges to be tackled, broadening task engagement and selfefficacy. Research has found that agreeableness can lead to increased self-efficacy. Certain researchers have found that individual selfefficacy is positively correlated with extraversion and negatively correlated with neuroticism (Schmitt, 2007). The finding of Djigić et al. (2014) indicated that conscientiousness predicted the self-efficacy of teachers, while Marcionetti and Rossier (2016) believed that conscientiousness, neuroticism, and extraversion were significantly correlated with self-efficacy. In sports, Wang et al. (2009) found that neuroticism had a significant negative predictive effect on the general selfefficacy of basketball players, while extraversion and conscientiousness had significant positive predictive effects.

Personality and self-control can be mediated with self-efficacy (Sikorska and Gerc, 2018). When considering the self-efficacy and selfregulation theories of Badura, a claim could be made that self-control affects self-efficacy and there exists a significant positive correlation between the two (Bandura, 1978). Au (2015) observed a positive correlation between selfefficacy and self-control. Additionally, there are studies focused on self-efficacy as a psychological factor influencing self-control performance. Baumeister's analysis emphasises that self-control requires an individual's own control resources, and self-efficacy is the complementary resource as it acts as a positive emotion (Baumeister, 2002). $\mathrm{Yu}$ and Yang (2008) suggest that an interaction effect exists between self-efficacy beliefs and selfcontrol behavior. Research indicates the positive effect of self-efficacy on self-control in athletes.

When considering the discussion above, these issues are subject to further research. We have knowledge of some features connected with achieving success, however, we still do not know how and in what combination it correlates with the genotype. Without any doubt, this remains an area for additional research, especially studies that will consider more psychological aspects and more elements in the genotype. For example, associations between dopamine D4 receptor gene polymorphisms and personality traits in elite athletes seem to be promising (MichałowskaSawczyn et al., 2019). Physical exercise elicits a widespread response in gene expression (Pacheco et al., 2018) and this could be intensity-dependent at least in skeletal muscle (Popov et al., 2018). The next step of our research is going to be the epigenetic aspect (methylation changes occurring in the domains of the DAT1 promoter) in correspondence with increased motivation among athletes, with special attention paid to those who achieved sport success at the highest level.

\section{Acknowledgements}

The study was supported by National Science Centre of Poland (No. UMO-2016/21/B/NZ7/01068).

\section{References}

$\mathrm{Au}$ EW. Locus of control, self-efficacy, and the mediating effect of outcome control: predicting course-level and global outcomes in an academic context. Anxiety Stress Coping, 2015; 28: 425-444

Bandura A. Self-efficacy: toward a unifying theory of behavioral change. Adv. Behav. Res. Ther, 1978; 1: 139161

Baumeister RF. Ego depletion and self-control failure: an energy model of the self 's executive function. Self Identity, 2002; 1: 129-136

Belsky J, Pluess M. Beyond diathesis stress: differentia susceptibility to environmental influences. Psychol Bull, 2009; 135: 885-908 
Bouchard T Jr. Genes environment and personality. Science, 1994; 264: 1700-1701

Brown SD, Lent RW, Telander K, Tramayne S. Social cognitive career theory, conscientiousness, and work performance: a meta-analytic path analysis. J. Vocat. Behav, 2011; 79: 81-90

Burke SM, van de Giessen E, de Win M, Schilt T, van Herk M, van den Brink W, Booij J. Serotonin and dopamine transporters in relation to neuropsychological functioning, personality traits and mood in young adult healthy subjects. Psychol Med, 2011; 41: 419-29

Carver CS, Connor-Smith J. Personality and coping. Ann Rev Psychol, 2010; 61: 679-704

Cherepkova EV, Maksimov VN, Kushnarev AP, Shakhamatov II, Aftanas Li. The polymorphism of dopamine receptor D4 (DRD4) and dopamine transporter (DAT) genes in the men with antisocial behavior and mixed martial arts fighters. World J Biol Psychiatry, 2019; 20(5): 402-415

De Vries RE, Van Gelder JL. Tales of two self-control scales: relations with five-factor and HEXACO traits. Personal. Individ. Differ, 2013; 54: 756-760

Djigić G, Stojiljković S, Dosković M. Basic personality dimensions and teachers' self-efficacy. Procedia. Soc. Behav. Sci, 2014; 112: 593-602

Dreher J, Kohn P, Kolachana B,Weinberger DR. Berman KF. Variation in dopamine genes influences responsivity of the human reward system. Proc Natl Acad Sci, 2009; 106: 617-622

Enter D, Colzato LS, Roelofs K. Dopamine transporter polymorphisms affect social approach-avoidance tendencies. Genes Brain Behav, 2012; 11: 671-676

Grace AA. Dysregulation of the dopamine system in the pathophysiology of schizophrenia and depression. Nat Rev Neurosci, 2016; 17: 524-532

Green JA, O'Connor DB, Gartland N, Roberts BW. The Chernyshenko conscientiousness scales: a new facet measure of conscientiousness. Assessment, 2016; 23: 374-385

Greenwood TA, Kelsoe JR. Promoter and intronic variants affect the transcriptional regulation of the human dopamine transporter gene. Genomics, 2003; 82: 511-520

Hughes S, Case HS, Stuempfle KJ, Evans D. Personality profiles of Iditasport ultra-marathon participants. J Appl Sport Psychol, 2003; 15: 256-261

Judge TA, Jackson CL, Shaw JC, Scott BA, Rich BL. Self-efficacy and work-related performance: the integral role of individual differences. J Appl Sport Psychol, 2007; 92: 107-127

Kawarai T, Kawakami H, Yamamura Y, Nakamura S. Structure and organization of the gene encoding human dopamine transporter. Gene, 1997; 195: 11-18

Krokosz D, Jochimek M. Coping strategies, perception of sport risk and satisfaction with life in men and women practicing extreme sports. Balt J Health Phys Activ, 2018; 10(4): 238-245

Marcionetti J, Rossier J. Global life satisfaction in adolescence: the role of personality traits, self-esteem, and self-efficacy. J. Individ. Differ, 2016; 37: 135-144

Michałowska-Sawczyn M, Niewczas M, Król P, Czarny W, Rzeszutko A, Chmielowiec K, Chmielowiec J, Grzywacz A, Humińska-Lisowska K, Lachowicz M, Trybek G, Kaczmarczyk M, Wilk M, Ficek K, Maculewicz E, Proia P, Cięszczyk P. Associations Between the Dopamine D4 Receptor Gene Polymorphisms and Personality Traits in Elite Athletes. Biol. Sport, 2019; 36: 365-372

McCrae RR, Costa Jr PT. Updating Norman's "Adequate Taxonomy": intelligence and personality dimensions in natural language and in questionnaires. J Pers Soc Psychol, 1985; 49: 710-721

Mill J, Asherson P, Browes C, D'Souza U, Craig I. Expression of the dopamine transporter gene is regulated by the 3_UTR VNTR: evidence from brain and lymphocytes using quantitative RT-PCR. Am. J. Med. Genet, 2002; 114: 975-979

Ozer D J, Benet-Martinez V. Personality and the prediction of consequential outcomes. Annual Review of Psychology, 2006; 57: 401-421

Pasini A, Sinibaldi L, Paloscia C, Douzgou S, Pitzianti MB, Romeo E, Curatolo P, Pizzuti A. Neurocognitive effects of methylphenidate on ADHD children with different DAT genotypes: a longitudinal open label trial. Eur. J. Paediatr. Neurol, 2013; 17: 407-414

Pacheco C, Felipe SMS, Soares MMDC, Alves JO, Soares PM, Leal-Cardoso JH, Loureiro ACC, Ferraz ASM, de Carvalho DP, Ceccatto VM. A compendium of physical exercise-related human genes: an 'omic scale analysis. Biol. Sport, 2018; 35: 3-11 
Popov DV, Makhnovskii PA, Kurochkina NS, Lysenko EA, Vepkhvadze TL, Vinogradova OL. Intensitydependent gene expression after aerobic exercise in endurance-trained skeletal muscle. Biol. Sport, 2018; 35: 277-289

Schmitt N. The interaction of neuroticism and gender and its impact on self-efficacy and performance. Hum. Perform, 2007; 21: 49-61

Sesack SR, Hawrylak VA, Matus C, Guido MA, Levey AI. Dopamine axon varicosities in the prelimbic division of the rat prefrontal cortex exhibit sparse immunoreactivity for the dopamine transporter. $J$ Neurosci, 1998; 18: 2697-2708

Sikorska I, Gerc K. Athletes with disability in the light of positive psychology. Balt J Health Phys Activ, 2018; 10(1): 64-76

Talmi D, Seymour B, Dayan P, Dolan RJ. Human Pavlovian-instrumental transfer. J Neurosci, 2008; 28: 360368

VanNess SH, Owens MJ, Kilts CD. The variable number of tandem repeats element in DAT1 regulates in vitro dopamine transporter density. BMC Genetics, 2005; 6: 55

Wang Z, Zhang W, Li YH, Zhang H, Hong P. The relationship between personality traits, self-efficacy and stress coping styles of woman basketball players. Chi. Sport. Sci, 2009; 29: 59-64

Yu GQ, Yang ZL. A research on the implicit effect of selfcontrol. Psychol. Sci, 2008; 31: 614-616

\section{Corresponding author:}

\section{Prof. dr hab. Paweł Cięszczyk}

Gdańsk University of Physical Education and Sport

ul. Kazimierza Górskiego 1

80-336 Gdańsk

E-mail: cieszczyk@poczta.onet.pl 